\title{
Optical bistability for two-level atoms in a standing-wave cavity
}

\author{
D. E. Grant and H. J. Kimble \\ Department of Physics, The University of Texas at Austin, Austin, Texas 78712
}

Received April 26, 1982

\begin{abstract}
Observations of optical bistability are reported for a system composed of multiple atomic beams passing through a high-finesse optical cavity. Both the transmitted power and the intracavity fluorescent intensity have been recorded as functions of incident laser power for zero cavity and atomic detunings. A quantitative study has been made of the evolution of the steady-state switching intensities from well below the critical onset of bistability to well above this point. The results show reasonable agreement with a Gaussian-beam theory of optical bistability, but systematic departures are noted.
\end{abstract}

Since the earliest work on optical bistability in a passive resonator, ${ }^{1-5}$ theoretical treatments of this subject have often dealt with an intracavity medium composed of two-level atoms. Although bistability has been demonstrated in other systems, and although a great deal of progress has been made in exploring this phenomenon, ${ }^{6,7}$ observations of bistability for the simple system of two-level atoms have for many years remained conspicuously absent from the literature. Quite recently important steps toward remedying this circumstance have been made with reports of bistability in systems that approximate a two-level structure. , $9^{9}$ In particular, Weyer et al. ${ }^{9}$ have observed absorptive bistability for an optical cavity containing multiple atomic beams in experiments in which effects that are due to Doppler broadening, optical pumping, and collisions were largely eliminated. Qualitative agreement between their results and those of a plane-wave model of optical bistability for two-level atoms was obtained.

We have likewise undertaken an investigation of optical bistability for two-level atoms in an atomic beam interacting with the intracavity field of a high-finesse resonator. ${ }^{10}$ In this Letter we describe our measurements, which we believe represent the first quantitative study of the evolution of the steady-state characteristics in absorptive optical bistability.

Our experimental arrangement consists of five primary atomic beams of sodium, each $0.5 \mathrm{~mm} \times 0.5 \mathrm{~mm}$ in cross section intersecting the axis of an optical resonator at $90^{\circ}$. The cavity is operated near the confocal spacing with $0.25-\mathrm{m}$ radius-of-curvature mirrors external to the vacuum chamber and is coupled to the atomic beams through windows with antireflection coatings. In the empty-cavity case (no atomic beams) and for a mode-matched incident laser beam, the finesse $\mathcal{F}$ of the cavity is $210 \pm 15$, the peak transmission coefficient $T$ is $0.018 \pm 0.003$, and the beam waist $\omega_{0}$ is approximately $150 \mu \mathrm{m}$. The small waist together with the relatively larger cross-sectional area of the atomic beams ensures that the intracavity medium appears as an extended medium transverse to the cavity axis. Along the cavity axis the beams are spread over $7 \mathrm{~mm}$.

In addition to the five primary atomic beams, a lack of adequate collimation produces two sets of secondary atomic beams, each symmetrically placed at an angle of roughly $\pm 8 \mathrm{mrad}$ relative to the primary beams. Both the primary and secondary atomic beams are collimated to approximately $\pm 2 \mathrm{mrad}$. Before entering the intracavity field of the resonator and in a region of uniform magnetic field along the cavity axis, the atomic beams are optically pumped with circularly polarized light to behave as two-level atoms in their subsequent interactions. ${ }^{11}$ The net result of this configuration of multiple atomic beams with optical pumping is to produce a single, symmetric absorption feature for the transition $3^{2} S_{1 / 2}, F=2, M_{F}=2 \rightarrow 3^{2} P_{3 / 2}, F=3, M_{F}$ $=3$ of $30-\mathrm{MHz}$ width (FWHM), compared with the natural linewidth of $10 \mathrm{MHz}$. By associating a Lorentzian profile of $14 \mathrm{MHz}$ FWHM with each set of primary and secondary beams, the measured line shape can be fitted reasonably well if the absorption peak of each set of secondary beams is offset by $\pm 13 \mathrm{MHz}$ and has a weight of 0.35 compared wtih the central primary beams. The maximum resonant absorption coefficient of the combined set of atomic beams is $\alpha l=1.5$, with roughly $15 \%$ of this value due to (off-resonant) secondary-beam absorption. Background absorption that is due to stray sodium vapor within the chamber is less than 0.005. The output of a single-mode dye laser stabilized in frequency to $0.25 \mathrm{MHz}$ rms serves as the excitation source for our measurements.

With atomic and cavity detunings near zero, we have recorded the input-output characteristics of the optical cavity plus atomic beams from an $x-y$ oscilloscope display of transmitted laser power $P_{T}$ and intracavity fluorescence $I_{F}$ as functions of incident laser power $P_{I}$. Figure 1 is a photograph obtained in this fashion and shows a fully developed hysteresis with clearly defined switching points from both the upper and lower branches. In Fig. 1 and for the other measurements reported here, the incident laser intensity was modulated sinusoidally at $100 \mathrm{~Hz}$. The corresponding time of $10 \mathrm{msec}$ taken to sweep through a hysteresis cycle is much longer than either the cavity-decay time $\left(5 \times 10^{-8}\right.$ $\mathrm{sec})$ or the atomic-relaxation time $\left(1.6 \times 10^{-8} \mathrm{sec}\right)$. Thus Fig. 1 is an observation of the steady-state characteristics in absorptive optical bistability and differs in this respect from the work of Weyer et al., ${ }^{9}$ whose 


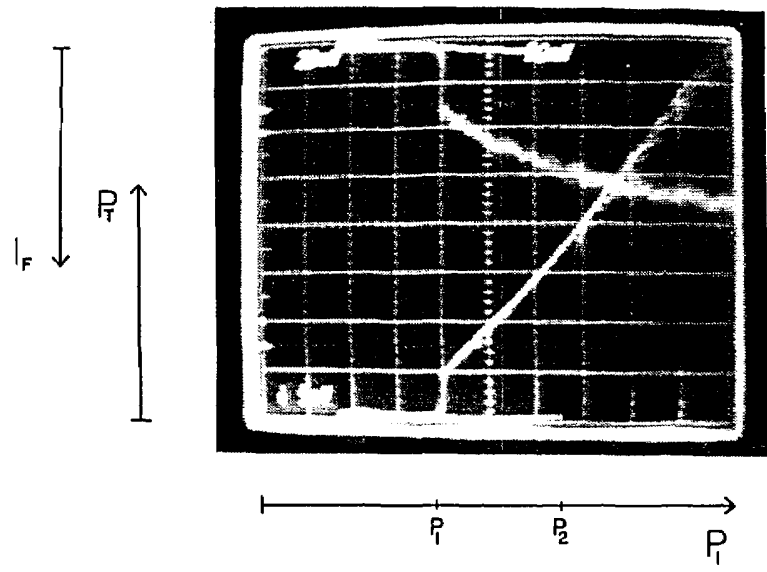

Fig. 1. Transmitted power $P_{T}$ (lower trace) and intracavity fluorescence $I_{F}$ (upper trace) as functions of incident laser power $P_{I}$ for zero atomic and cavity detunings. Note that $I_{F}$ increases in a downward direction. The resonant atomicabsorption coefficient $\alpha l$ is 1.0, and the incident switching powers $P_{1}$ and $P_{2}$ are 0.63 and $1.0 \mathrm{~mW}$, respectively.

data were obtained in a transient regime for fast sweep times ( $6 \mu \mathrm{sec})$. The switching times recorded in Fig. 1 are detector limited.

In order to compare our results with one of the various theories of the deterministic regime in optical bistability, we must adopt a treatment that incorporates the effects of inhomogeneous broadening, standing waves, and transverse modes. $\mathrm{McCall}^{2}$ and Drummond ${ }^{12}$ have considered each of these effects; Ballagh et al. ${ }^{13}$ have dealt with the latter two. These theories should be applicable to our cavity because of its high finesse and efficient diffractive mixing. Following the work of Drummond, ${ }^{12}$ we evaluate the cooperativity parameter $C$ from a measurement of the atomic-absorption coefficient $\alpha l$ and the normalized switching intensities $Y_{1,2}$ from the incident switching powers $P_{1,2}$ by introducing the definitions

$$
\begin{aligned}
& C \equiv \frac{\alpha l F}{2 \pi}, \\
& Y \equiv \frac{3 P_{I}}{\pi \omega_{0}^{2} I_{S}} \epsilon .
\end{aligned}
$$

Here $\mathcal{F}, \alpha l, \omega_{0}$, and $P_{I}$ are as previously defined and $I_{S}$ is the saturation intensity $\left[=6.1\left(\mathrm{~mW} / \mathrm{cm}^{2}\right)\right]$. The factor $\epsilon$ expresses the enhancement in intracavity intensity relative to incident intensity for the cavity without atomic beams. For an ideal cavity, $\epsilon$ would equal $1 /(1-R)$, with $R$ the mirror reflectivity. For our cavity we choose

$$
\epsilon=\frac{\sqrt{\mathcal{T}}}{\pi} \mathcal{F}
$$

with $\mathcal{T}$ the transmission coefficient for zero detuning for the empty cavity (no atomic beams). The definitions [Eqs. (1)-(3)] take into account the nonzero absorption losses in the mirror coatings of the cavity and the small linear losses suffered within the cavity, as, for example, at the surfaces of the antireflection-coated windows of the vacuum chamber. In the case of Eqs.
(2) and (3), Drummond's variable $Y$ has been modified by the simple prescription that $(1-R)$ is replaced by $T$, with $T$ the mirror transmittance. ${ }^{12}$

From a large number of photographs such as that shown in Fig. 1, and over a wide range of intracavity atomic density, we have recorded the dependence of the switching powers $\left(P_{1}, P_{2}\right)$ on the atomic-absorption coefficient $\alpha l$, again for zero atomic and cavity detunings. The result of these measurements is shown in Fig. 2 , in which we see that below some critical atomic density bistability is not observed. However, at higher atomic-beam densities a hysteresis cycle emerges and gradually increases in size, as indicated by two points in $Y$ for a given $C>C_{\text {critical }}$.

In Fig. 2 we have computed for each set of incident switching powers $\left(P_{1}, P_{2}\right)$ a value of the atomic-cooperativity parameter $C$ from Eq. (1) directly from our measurements of $\alpha l$ and $\mathcal{F}$. This procedure leads to an overall uncertainty of $\pm 15 \%$ for the scale of $C$ for the data in Fig. 2; however, in plotting this figure no adjustment of $C$ within this range has been made. Apart from the question of scaling, the relative uncertainty of points within the set is indicated by horizontal error bars at several points. In a similar fashion, the determination from Eqs. (2) and (3) of $\left(Y_{1}, Y_{2}\right)$ from the measured powers $\left(P_{1}, P_{2}\right)$ involves a factor uncertain to $\pm 30 \%$ based on the precision to which $\mathcal{F}, \mathcal{T}$, and $\omega_{0}$

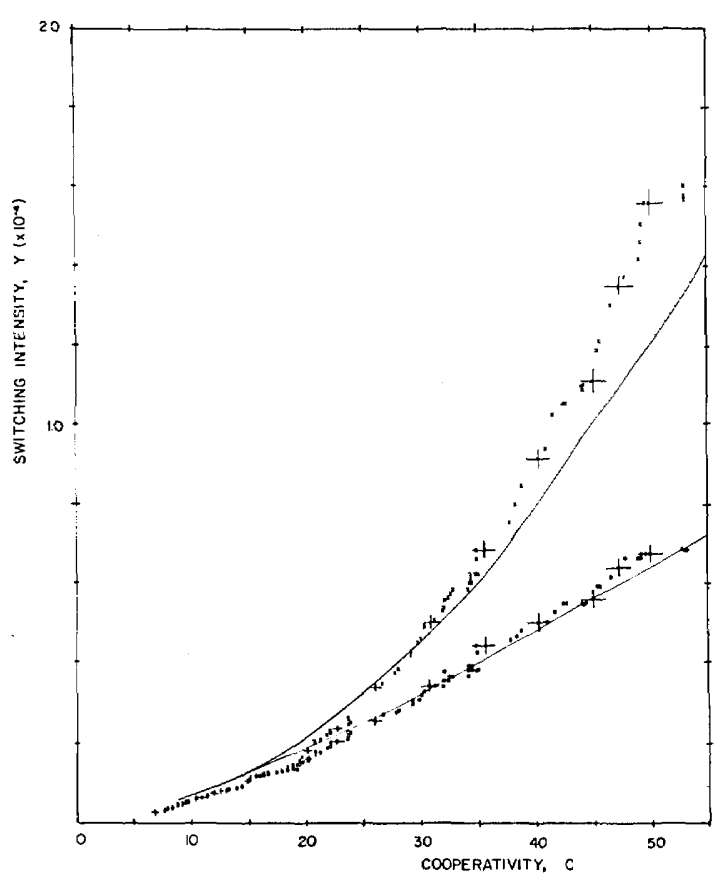

Fig. 2. Normalized incident switching intensities $Y$ versus atomic cooperativity $C$ for zero atomic and cavity detunings. Switching from the lower to upper branch of the hysteresis cycle is indicated by crosses; from the upper to lower branch, by open circles. A filled circle corresponds to the intensity of largest differential gain in the input-output characteristics before the onset of bistability. Each set of points has been obtained from a photograph such as that in Fig. 1. Relative uncertainties are indicated at several points. The determination of $Y$ and $C$ for the data is discussed in the text. The full curve is the theoretical result of Drummond ${ }^{12}$ with Gaussian inhomogeneous broadening. 


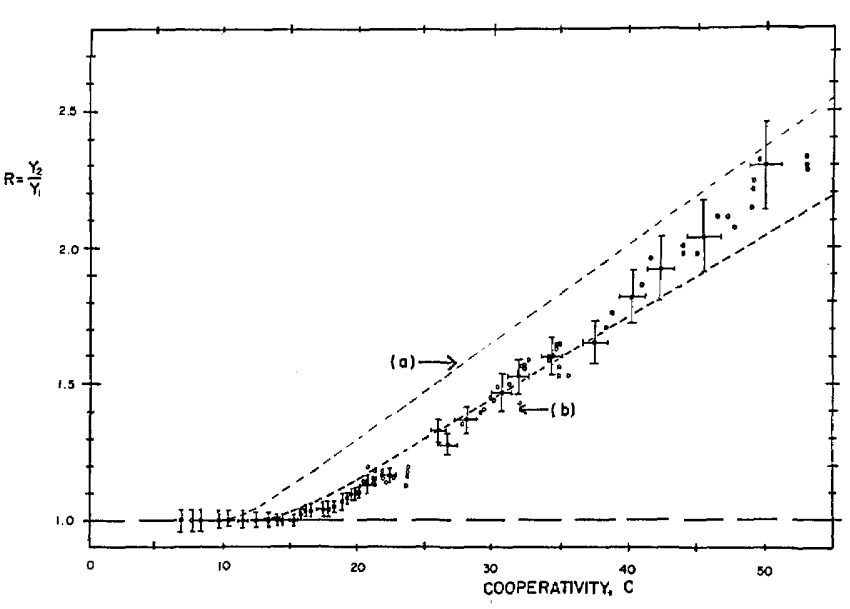

Fig. 3. From the same data set as in Fig. 2, the ratio $R=$ $Y_{2} / Y_{1}$ of incident switching intensities is plotted as a function of atomic cooperativity $C$. Below a critical value of $C$, bistability is not observed, and $R=1.0$. The dashed lines are from Drummond ${ }^{12}$ for (a) the case of homogeneous broadening and (b) Gaussian inhomogeneous broadening resulting in a total absorption width of $30 \mathrm{MHz}$.

are known. The values for $Y$ for the data in the figure have been calculated from the measured quantities $P_{1,2}$, $\omega_{0}, \mathcal{F}$, and $\mathcal{T}$ and then reduced by a factor of $f=1.8 \mathrm{in}$ order to optimize the comparison with theory. Relative uncertainties in the determination of $\left(P_{1}, P_{2}\right)$ are indicated by the vertical error bars.

The full curve shown in Fig. 2 is from the work of Drummond, ${ }^{12}$ taking into account inhomogeneous broadening. The curve is obtained from a calculation of the turning points of the state equation for Gaussian inhomogeneous broadening of the same magnitude present in our experiment although, as previously discussed, the inhomogeneity in the experiment is not due to simple Gaussian broadening. Because of this we cannot say with confidence that the factor of $1.8 \mathrm{re}-$ quired to bring the experimental switching points into rough agreement with the theoretical curve indicates a basic disparity between experiment and theory. Furthermore, most experimental uncertainties, e.g., slight misalignments of the cavity or a lack of precise mode matching to the cavity, tend to conspire to increase the observed switching powers relative to the theory.

To circumvent these questions regarding the scale of $Y$, we have plotted in Fig. 3 the ratio $R$ of switching powers $P_{2} / P_{1}\left(=Y_{2} / Y_{1}\right)$ as a function of the cooperativity parameter $C$. The dashed lines labeled (a) and (b) in Fig. 3 are again from Drummond ${ }^{12}$ and are, respectively, for no inhomogeneous broadening and for Gaussian inhomogeneous broadening resulting in a total absorption width of $30 \mathrm{MHz}$ (as in Fig. 2). Curve (b) agrees reasonably well with our data in the region of the onset of bistability, especially with regard to the substantially higher value of $C_{\text {critical }} \simeq 14$, compared with the prediction of theories that do not include transverse or standing-wave effects. At larger values of $C>$
$C_{\text {critical, a systematic departure between theory and }}$ experiment appears, with the observed hysteresis evolving faster with increasing $C$ than either of the results (a) or (b). Although we do not at present have an explanation for this departure, we do not believe that a simple rescaling of $C$ within the experimental uncertainty would improve matters since then the fit in the threshold region would be degraded.

In conclusion, we have reported observations of absorptive optical bistability in a system of atomic beams within a standing-wave cavity. A quantitative comparison with a Gaussian-beam model of optical bistability has been made, producing reasonable overall agreement for the evolution of the steady-state switching intensities as a function of the cooperativity parameter $C$. Systematic departures have been noted and are the subject of a continuing investigation.

P. D. Drummond provided the theoretical results shown in Figs. 2 and 3 . Conversations with him have been most stimulating and rewarding. The technical support of L. Deavers has helped to make these experiments possible. The support and interest in this research of M. Fink and W. Schieve is gratefully acknowledged.

This work was initiated with funds provided by The University of Texas at Austin and by the Research Corporation. Continuing support is provided in part by the Joint Services Electronics Program and by the Robert A. Welch Foundation.

\section{References}

1. H. Seidel, U.S. Patent No. 3,610,731 (October 5,1971); A. Szöke, V. Daneu, J. Goldhar, and N. A. Kurnit, Appl. Phys. Lett. 15, 376 (1969); J. W. Austin and L. G. DeShazer, J. Opt. Soc. Am. 61, 650 (1971); E. Spiller, J. Opt. Soc. Am. 61, 669 (1971), J. Appl. Phys. 43, 1673 (1972).

2. S. L. McCall, Phys. Rev. A 9, 1515 (1974).

3. H. M. Gibbs, S. L. McCall, and T. N. C. Venkatesan, Phys. Rev. Lett. 36, 1135 (1976).

4. R. Bonifacio and L. A. Lugiato, Opt. Commun. 19, 172 (1976).

5. For a review of the theoretical and experimental work in this field, see the article by H. M. Gibbs, S. L. McCall, and T. N. C. Venkatesan, Opt. News 5(3), 6-12 (1979).

6. C. M. Bowden, M. Ciftan, and H. R. Robl, eds., Optical Bistability (Plenum, New York, 1981).

7. P. W. Smith, ed., IEEE J. Quantum Electron., Special Issue on Optical Bistability, QE-17 (1981).

8. W. J. Sandle and A. Gallagher, Phys. Rev. A 24, 2017 (1981).

9. K. G. Weyer, H. Widenmann, M. Rateike, W. R. MacGillivray, P. Meystre, and H. Walther, Opt. Commun. 37, 426 (1981).

10. H. J. Kimble and D. E. Grant, J. Opt. Soc. Am. 12, 1639(A) (1981).

11. J. A. Abate, Opt. Commun. 10, 269 (1974).

12. P. D. Drummond, IEEE J. Quantum Electron. QE-17, 301 (1981).

13. R. J. Ballagh, J. Cooper, M. W. Hamilton, W. J. Sandle, and D. M. Warrington, Opt. Commun. 37, 143 (1981). 